\title{
Infrared Variability of Two Dusty White Dwarfs
}

\author{
Siyi Xu (许偲艺) $)^{1}$ (1) , Kate Y. L. Su ${ }^{2}$ (1) , L. K. Rogers ${ }^{3}$, Amy Bonsor $^{3}$, Johan Olofsson $^{4,5}$ (1) , Dimitri Veras ${ }^{6,7,12}$ (1), \\ Rik van Lieshout ${ }^{3}$, Patrick Dufour ${ }^{8}$, Elizabeth M. Green ${ }^{2}$ (D), Everett Schlawin ${ }^{2}$ (D), Jay Farihi ${ }^{9}$, Thomas G. Wilson ${ }^{9,10}$ (iD, \\ David J. Wilson $^{7,11}$ (D), and Boris T. Gänsicke ${ }^{7}$ (D) \\ ${ }^{1}$ Gemini Observatory, 670 N. A’ohoku Place, Hilo, HI 96720, USA; sxu@gemini.edu \\ ${ }^{2}$ Steward Observatory, University of Arizona, 933 N. Cherry Avenue, Tucson, AZ 85721, USA \\ ${ }^{3}$ Institute of Astronomy, University of Cambridge, Madingley Road, Cambridge CB3 0HA, UK \\ ${ }^{4}$ Instituto de Física y Astronomía, Facultad de Ciencias, Universidad de Valparaíso, Av. Gran Bretaña 1111, Playa Ancha, Valparaíso, Chile \\ ${ }^{5}$ Núcleo Milenio Formación Planetaria—NPF, Universidad de Valparaíso, Av. Gran Bretaña 1111, Valparaíso, Chile \\ ${ }^{6}$ Centre for Exoplanets and Habitability, University of Warwick, Coventry CV4 7AL, UK \\ ${ }^{7}$ Department of Physics, University of Warwick, Coventry CV4 7AL, UK \\ ${ }^{8}$ Institut de Recherche sur les Exoplanètes (iREx), Université de Montréal, Montréal, QC H3C 3J7, Canada \\ ${ }^{9}$ Department of Physics and Astronomy, University College London, London WC1E 6BT, UK \\ ${ }^{10}$ Isaac Newton Group of Telescopes, E-38700 Santa Cruz de La Palma, Spain \\ ${ }^{11}$ McDonald Observatory, C1400, University of Texas at Austin, Austin, TX 78712, USA \\ Received 2018 July 8; revised 2018 August 24; accepted 2018 August 24; published 2018 October 17
}

\begin{abstract}
The most heavily polluted white dwarfs often show excess infrared radiation from circumstellar dust disks, which are modeled as a result of tidal disruption of extrasolar minor planets. Interaction of dust, gas, and disintegrating objects can all contribute to the dynamical evolution of these dust disks. Here, we report two infrared variable dusty white dwarfs, SDSS J1228+1040 and G29-38. For SDSS J1228+1040, compared to the first measurements in 2007, the IRAC [3.6] and [4.5] fluxes decreased by $20 \%$ before 2014 to a level also seen in the recent 2018 observations. For G29-38, the infrared flux of the $10 \mu \mathrm{m}$ silicate emission feature became $10 \%$ stronger between 2004 and 2007, We explore several scenarios that could account for these changes, including tidal disruption events, perturbation from a companion, and runaway accretion. No satisfactory causes are found for the flux drop in SDSS J1228+1040 due to the limited time coverage. Continuous tidal disruption of small planetesimals could increase the mass of small grains and concurrently change the strength of the $10 \mu \mathrm{m}$ feature of G29-38. Dust disks around white dwarfs are actively evolving and we speculate that there could be different mechanisms responsible for the temporal changes of these disks.
\end{abstract}

Key words: circumstellar matter - minor planets, asteroids: general - stars: individual (G29-38, SDSS J122859.93 $+104032.9)$ - white dwarfs

\section{Introduction}

G29-38 was the first single white dwarf discovered to display excess infrared emission (Zuckerman \& Becklin 1987), and follow-up studies have shown that the excess flux arises from a close-in hot dust disk (Graham et al. 1990). The origin of such a dust disk remained a mystery until the asteroid tidal disruption model was proposed (Debes \& Sigurdsson 2002; Jura 2003). According to this model, the disks are remnants of minor planets that were perturbed into the tidal radius of the white dwarf and eventually became totally disrupted. The infrared excess is often modeled as a geometrically thin and optically thick disk within the tidal radius of the white dwarf (Jura 2003). These compact hot dust disks (temperature $\sim 1000 \mathrm{~K}$, size $0.01 \mathrm{au}$ ) around white dwarfs are morphologically different from debris disks around main-sequence stars (temperature $\sim 100 \mathrm{~K}$, size a few tens to hundreds of astronomical units). There are more than 40 white dwarfs that show infrared excess emission consistent with the presence of dusty disks (Farihi 2016). Some of the dusty white dwarfs also display calcium triplet emission from circumstellar gas that spatially coincides with the dust disk (e.g., Gänsicke et al. 2006, 2008).

About $25 \%-50 \%$ of white dwarfs are polluted-they display elements heavier than helium in their spectra (Zuckerman et al. 2003, 2010; Koester et al. 2014). In many cases,

\footnotetext{
${ }^{12}$ STFC Ernest Rutherford Fellow.
}

continuous accretion onto the white dwarf from circumstellar material is needed due to the short settling times of heavy elements. The connection between atmospheric pollution and dust disks was first explored in von Hippel et al. (2007). The most heavily polluted white dwarfs are often accompanied by an infrared excess from a dust disk. Spectroscopic observations of these polluted atmospheres have opened up a new field of measuring chemical compositions of extrasolar planetary material (Jura \& Young 2014; Harrison et al. 2018; Hollands et al. 2018).

Some polluted white dwarfs are dynamically active and they vary on short timescales. For example, the infrared flux of SDSS J0959-0200 dropped by 35\% between two observations in 2010 and then remained at the same level until at least 2014 (Xu \& Jura 2014). The gas emission lines around WD J1617 +1620 disappeared within a few years (Wilson et al. 2014). Most gas disks show gradual variations over a few years (Wilson et al. 2015; Manser et al. 2016a, 2016b; Redfield et al. 2017; Dennihy et al. 2018). Recently, transits from an actively disintegrating asteroid were detected around WD 1145 +017 (Vanderburg et al. 2015) - a white dwarf that is also heavily polluted, has an infrared excess from a dust disk, and displays absorption lines from circumstellar gas ( $\mathrm{Xu}$ et al. 2016). The optical light curve of WD $1145+017$ is changing on a daily basis, likely due to the vigorous nature of tidal disruption (e.g., Gänsicke et al. 2016; Gary et al. 2017). 
Table 1

White Dwarf Parameters

\begin{tabular}{lcc}
\hline \hline & SDSS J1228+1040 & $\mathrm{G} 29-38$ \\
\hline$T_{\text {eff }}(\mathrm{K})$ & 23510 & 11240 \\
$\log g(\mathrm{cgs})$ & 8.16 & 8.00 \\
Dom. $^{\mathrm{a}}$ & $\mathrm{H}$ & $\mathrm{H}$ \\
$M_{\mathrm{wd}}\left(M_{\odot}\right)$ & 0.70 & 0.59 \\
$R_{\mathrm{wd}}\left(R_{\odot}\right)$ & 0.012 & 0.013 \\
$d(\mathrm{pc})^{\mathrm{b}}$ & 127 & 17.5 \\
$V(\mathrm{mag})$ & 16.2 & 13.0 \\
$\operatorname{Ref}$ & Tremblay et al. (2011) & Subasavage et al. (2017) \\
\hline
\end{tabular}

Notes.

a Dominant element in the white dwarf's atmosphere.

b Distance is taken from Gaia DR2 (Gaia Collaboration et al. 2018).

The dynamical mechanism responsible for tidal disruption and white dwarf pollution is an area of active research (e.g., Veras 2016). The general consensus is that minor planets (i.e., asteroids, comets) and giant planets beyond a few astronomical units can survive the post-main-sequence evolution and orbit around white dwarfs (Nordhaus \& Spiegel 2013; Mustill et al. 2014). Through different dynamical interactions, e.g., mean motion resonance, planet-planet scattering, secular resonance sweeping, and the Kozai-Lidov effect, the orbits of these minor planets are perturbed-some are ejected from the system while others can enter into the white dwarf's tidal radius $\left(\sim 100 R_{\mathrm{wd}}\right.$, Debes et al. 2012; Stephan et al. 2017; Mustill et al. 2018; Smallwood et al. 2018). In addition, there is evidence for continuous accretion of small planetesimals (Wyatt et al. 2014).

In this paper, we focus on two systems, SDSS J1228+1040 and G29-38. Their basic parameters are listed in Table 1. SDSS J1228+1040 is the prototype of white dwarfs with circumstellar gas debris (Gänsicke et al. 2006). Its infrared excess was reported in Brinkworth et al. (2009). Through 12 years of optical spectroscopic monitoring, Manser et al. (2016b) found a gradual variation of the gas emission lines and they proposed that it was the result of precession of an asymmetric pattern under general relativity. SDSS J1228 +1040 is also heavily polluted and the composition of the accreting material resembles that of bulk Earth (Gänsicke et al. 2012).

G29-38 was the first white dwarf discovered to have an infrared excess and a $10 \mu \mathrm{m}$ silicate emission feature (Reach et al. 2005, 2009). The star also has a polluted atmosphere (Koester et al. 1997) and recent HST/COS observations show that it is accreting from volatile-poor material that is similar in composition to bulk Earth (Xu et al. 2014). G29-38 is among the first optically variable white dwarfs discovered (Shulov \& Kopatskaya 1974; McGraw \& Robinson 1975). The newest addition to the wonders of G29-38 comes from the discovery of molecular hydrogen in its atmosphere, which provides an additional constraint of its stellar parameters (Xu et al. 2013). It is among the hottest stellar environments with a molecular hydrogen detection.

Here, we report infrared observations of the dust disks around SDSS J1228+1040 and G29-38, demonstrating for the first time that these two disks are intrinsically variable. The rest of the paper is organized as follows. Observation and data reduction are presented in Section 2. In Section 3, we present some disk models that could explain the temporal variations of the infrared luminosity. Possible scenarios are explored in Section 4 and results are summarized in Section 5.

\section{Observation and Data Reduction}

\subsection{Spitzer/IRAC and MIPS Observations}

SDSS J1228+1040 and G29-38 have been observed multiple times with Spitzer/IRAC (Fazio et al. 2004), as summarized in Tables 2 and 3. Both stars are well detected in each frame and separated from background stars. We performed aperture photometry on individual artifact-corrected CBCD (Corrected Basic Calibrated Data) frames with the IDL programs BOX CENTROIDER.PRO and APER.PRO. An aperture radius of 3 native pixels (3!"6) and a sky annulus of 12-20 pixels (14". 4-24".0) were used for the analysis. Aperture correction, pixel phase, and array location correction were also performed. For each epoch, we report the average flux weighted by the signal-to-noise ratio of each measurement and take the weighted uncertainty as the final uncertainty. Since we are interested in relative flux difference, our reported numbers only include measurement uncertainty. As an additional check, we also performed aperture photometry on the combined mosaic and obtained similar results.

For SDSS J1228+1040, we found significant variability in the first two Spitzer observations and the flux levels have remained the same in the third epoch, as shown in Figure 1. The infrared fluxes have dropped by $20 \%$ at $3 \sigma$ significance at both [3.6] and [4.5]. For the background stars of similar brightness, we found that their fluxes agree to within $1 \%$ for both [3.6] and [4.5] at all three epochs. The relative flux drop of SDSS J1228+1040 between 2007 and 2014 is detected at high significance.

For G29-38, there is some dispersion in the flux level in the individual CBCD frames, as indicated by the spread of the gray dots in Figure 1. The maximum flux difference between different epochs/AORs is 3.3\% $(1.2 \sigma)$ at [3.6], 6.0\% $(2.1 \sigma)$ at [4.5], 2.0\% $(0.6 \sigma)$ at [5.8], and 7.7\% $(2.8 \sigma)$ at [8.0]. As a sanity check, we performed aperture photometry on a few background stars and found that their fluxes agree within $2 \%, 2 \%, 6 \%$, and $7 \%$ for [3.6], [4.5], [5.8], and [8.0] respectively. G29-38 is the brightest star in the field of view and yet its photometry stability is worse compared to the faint field stars, particularly at [3.6] and [4.5]. This is expected because the exposure time is $30 \mathrm{~s}$, which is much shorter than the pulsation periods (typically tens of minutes, see Kleinman et al. 1998). We defer the discussion of pulsation-induced flux variation to Section 3.2.1.

Spitzer/MIPS observations (Rieke et al. 2004) of SDSS J1228+1040 and G29-38 have been published by using early Spitzer pipelines and calibrations (Brinkworth et al. 2009; Reach et al. 2009; Farihi et al. 2014). Here, we reprocessed all the data using the MIPS instrument in-house pipeline with the final calibration established for the mission (described in Sierchio et al. 2014). SDSS J1228+1040 has three deep $24 \mu \mathrm{m}$ observations with a total exposure time of $600 \mathrm{~s}$. The source is weakly detected in the individual mosaic. G29-38 is a clean point-like source at $24 \mu \mathrm{m}$ but not detected at $70 \mu \mathrm{m}$. We measure the photometry from the mosaics, and then adopt the average and weighted uncertainty as the final flux and the uncertainty, respectively, as reported in Table 2. 
Table 2

Spitzer Fluxes of SDSS J1228+1040

\begin{tabular}{lccccccccc}
\hline \hline UT Date & MJD & PID & AORKEY & $\begin{array}{c}\text { Time } \\
(\mathrm{s})\end{array}$ & $\begin{array}{c}3.6 \mu \mathrm{m} \\
(\mu \mathrm{Jy})\end{array}$ & $\begin{array}{c}4.5 \mu \mathrm{m} \\
(\mu \mathrm{Jy})\end{array}$ & $\begin{array}{c}5.8 \mu \mathrm{m} \\
(\mu \mathrm{Jy})\end{array}$ & $\begin{array}{c}8.0 \mu \mathrm{m} \\
(\mu \mathrm{Jy})\end{array}$ & $\begin{array}{c}\text { MIPS } \\
(\mu \mathrm{Jy})\end{array}$ \\
\hline 2007 Jun 30 & 53281.3 & 40048 & 22247936 & $100 \times 10$ & $228 \pm 10$ & $235 \pm 12$ & $225 \pm 12$ & $246 \pm 31$ & $\ldots$ \\
2014 Sep 3 & 56903.4 & 10032 & 49253376 & $30 \times 30$ & $184 \pm 9$ & $188 \pm 8$ & $\ldots$ & $\ldots$ & $\ldots$ \\
2018 May 9 & 58247.6 & 13216 & 64912384 & $12 \times 10$ & $180 \pm 9$ & $195 \pm 10$ & $\ldots$ & $\ldots$ & $\ldots$ \\
2008 Jul 25 & 54672.1 & 50118 & $25459712,25459456,25459200$ & $10 \times 60$ & $\ldots$ & $\ldots$ & $\ldots$ & $\ldots$ & $24 \mu \mathrm{m}: 129 \pm 10$ \\
\hline
\end{tabular}

\subsection{WISE Observations}

Since the launch of the Wide-field Infrared Survey Explorer (WISE; Wright et al. 2010) in 2010, SDSS J1228+1040 and G29-38 have been observed by WISE and now NEOWISE (Mainzer et al. 2011) every half year. Because they are faint, these stars are only detected in the two shortest bands in WISE, which have similar bandwidths as IRAC [3.6] and [4.5]. By using the NASA/IPAC infrared science archive, we extracted the photometry from the multiepoch photometry table and the Single Exposure Source Table for WISE and NEOWISE, respectively. We calculated the weighted average flux for all observations taken within 10 days, as plotted in Figure 1. For SDSS J1228+1040, the uncertainties are too large to detect the $20 \%$ flux drop identified in IRAC. For G29-38, similar to the IRAC observations, the WISE data show that G29-38 is not photometrically stable and there are low level variabilities from pulsation.

\subsection{Spitzer/IRS Spectroscopy}

G29-38 has also been observed with the Spitzer/IRS instrument (Houck et al. 2004) during the cryogenic mission: 2004 December 8 (AORKEY 10184192), 2006 June 30 (AORKEY 13828096), and 2007 August 4 (AORKEY 22957568). The Short-Low (SL, 5.2-14.5 $\mu \mathrm{m}$, resolution 100) module was used in both 2004 and 2007, while the SL2 $(5.2-8.7 \mu \mathrm{m})$ and Long-Low (LL, 19.4-38 $\mu \mathrm{m}$, resolution 100) modules were used in 2006. The 2004 and 2006 IRS spectra that were reduced by an early version of the pipeline were published by Reach et al. (2009). For this study, we used the spectra from the CASSIS website that provides uniform, high-quality IRS spectra optimally extracted for point-like sources (Lebouteiller et al. 2011).

As shown in Figure 2, the flux of G29-38 in the 5-7 $\mu \mathrm{m}$ region agrees within $2 \%$ over the three-year period, but the 2007 flux in the $10 \mu \mathrm{m}$ silicate feature region increased by $10 \%$. The temporal variability in the IRS spectra is in line with the IRAC photometry presented in Section 2.1. To make a direct comparison, we computed the synthesized [8.0] photometry using the observed IRS spectra: $8.14 \mathrm{mJy}$ in 2004 and $8.56 \mathrm{mJy}$ in 2007, consistent with the 5\% increase in the [8.0] photometry from 2004 to 2007 (see Table 3). Given that the 5-7 $\mu \mathrm{m}$ IRS flux between the 2004 and 2007 epochs agrees within $2 \%$, we conclude that the temporal variability between 2004 and 2007 IRS spectra is significant.

\subsection{UKIRT JHK Observations}

Since 2015, we have been monitoring dusty white dwarfs with the UK Infrared Telescope (UKIRT) to understand the origin of their variability. Details of the survey strategy and data reduction will be presented in L. K. Rogers et al. (2018, in preparation). For SDSS J1228+1040 and G29-38, the raw data were processed with pipelines produced by the Cambridge Astronomical Survey Unit (CASU). We compare the standard deviation of each frame and the uncertainty of the average flux and take the larger of the two values as the measurement uncertainty, as listed in Tables 4 and 5. Calibration uncertainties are not considered here. For SDSS J1228+1040, we detected a $13 \%(2.7 \sigma)$ drop in the $\mathrm{K}$ band flux between 2007 and 2015 and it has remained at the same level since then. As shown in Figure 1, this $\mathrm{K}$ band flux drop is likely related to the IRAC flux change. For G29-38, there is a 7\% (3.7 $\sigma)$ variation in the $\mathrm{K}$ band and it is consistent with pulsation-induced infrared variation (see Section 3.2.1).

\subsection{Optical Photometric Monitoring}

SDSS J1228+1040 was observed on 2018 March 21 (UT) using the University of Arizona's 61 inch (1.55 m) Kuiper telescope on Mt. Bigelow, Arizona. The camera was equipped with the Mont4k CCD, binned $3 \times 3$ to 0 ". 43 per pixel. The Schott-8612 filter (a broad white-light filter) was used. We adopted an exposure time of $30 \mathrm{~s}$, resulting in a cadence of $44.8 \mathrm{~s}$ including setup and readout. Conditions during the observation were nearly photometric and moonless. We obtained a continuous $6 \mathrm{hr}$ observations of SDSS J1228 +1040 and reached a typical S/N of 300 per frame.

All the images were bias-subtracted, flat-fielded, and bad pixel-cleaned in the usual manner. Aperture photometry was performed using the task PHOT in the IRAF DAOPHOT package. We performed relative photometry by referencing eight nearby stars within the $10^{\prime} \times 10^{\prime}$ field of view. After correcting the color response due to the airmass between our target (blue star) and field stars (generally red stars), we found no significant optical variability with a standard deviation of 0.006 mag (see Figure 3).

\section{Disk Modeling}

\subsection{SDSS J1228+1040: Opaque Disk}

The white dwarf SDSS J1228+1040 is stable to 0.006 mag at optical wavelengths (see Section 2.5) and, therefore, the infrared variability must come from the circumstellar material. In terms of a simple geometrically thin and optically thick disk model (Jura 2003), we assume that the gas and dust occupy a similar region, and that sublimation and/or collisions of dust particles feed material into the gas disk, which then get accreted by the star. Its circumstellar gaseous disk is inferred to have an inclination of $70^{\circ}$, a small eccentricity, and a radius of 60-120 $R_{\mathrm{wd}}$ (Gänsicke et al. 2006). To minimize the number of free parameters, we take the disk eccentricity to be 0 . There are three free parameters, inner disk radius $R_{\text {in }}$, outer disk radius $R_{\text {out }}$, and line-of-sight disk inclination $i$. The white dwarf flux is calculated as with parameters 
Table 3

Spitzer Fluxes of G29-38

\begin{tabular}{|c|c|c|c|c|c|c|c|c|c|}
\hline UT Date & MJD & PID & AORKEY & $\begin{array}{c}\text { Time }^{\mathrm{a}} \\
(\mathrm{s})\end{array}$ & $\begin{array}{c}3.6 \mu \mathrm{m} \\
(\mathrm{mJy})\end{array}$ & $\begin{array}{c}4.5 \mu \mathrm{m} \\
(\mathrm{mJy})\end{array}$ & $\begin{array}{c}5.8 \mu \mathrm{m} \\
(\mathrm{mJy})\end{array}$ & $\begin{array}{c}8.0 \mu \mathrm{m} \\
(\mathrm{mJy})\end{array}$ & $\begin{array}{l}\text { MIPS } \\
(\mathrm{mJy})\end{array}$ \\
\hline 2004 Nov 26 & 53335.5 & 2313,3548 & 10119424,11124224 & $30 \times 20 / 26^{b}$ & $8.11 \pm 0.12$ & $8.85 \pm 0.10$ & $8.22 \pm 0.12$ & $8.20 \pm 0.10$ & $\cdots$ \\
\hline 2005 Dec 22 & 53727.0 & 20026 & 13835264 & $12 \times 10$ & $7.91 \pm 0.17$ & $\ldots$ & $8.28 \pm 0.23$ & $\ldots$ & $\ldots$ \\
\hline 2007 Dec 26 & 54460.5 & 40369 & 22957312 & $30 \times 5$ & $8.01 \pm 0.22$ & $8.89 \pm 0.22$ & $8.13 \pm 0.22$ & $8.38 \pm 0.20$ & $\ldots$ \\
\hline 2007 Dec 28 & 54462.2 & 40369 & 22960896 & $30 \times 5$ & $8.17 \pm 0.23$ & $8.99 \pm 0.22$ & $8.25 \pm 0.23$ & $8.55 \pm 0.20$ & $\ldots$ \\
\hline 2007 Dec 31 & 54465.5 & 40369 & 22961152 & $30 \times 5$ & $8.35 \pm 0.23$ & $8.98 \pm 0.22$ & $8.44 \pm 0.23$ & $8.48 \pm 0.20$ & $\ldots$ \\
\hline 2008 Jul 18 & 54665.7 & 40369 & 22961408 & $30 \times 6$ & $8.04 \pm 0.22$ & $9.38 \pm 0.22$ & $8.38 \pm 0.23$ & $8.84 \pm 0.21$ & $\ldots$ \\
\hline 2009 Aug 19 & 55062.6 & 60161 & 35008512 & $30 \times 21$ & $\cdots$ & $8.99 \pm 0.12$ & $\ldots$ & $\cdots$ & $\cdots$ \\
\hline 2004 Dec 2 & 53341.2 & 2313 & 10149376 & $10 \times 3$ & $\ldots$ & $\ldots$ & $\ldots$ & $\ldots$ & $24 \mu \mathrm{m}: 2.75 \pm 0.05$ \\
\hline 2008 Jul 28 & 54675.3 & 50401 & 26134016 & $10 \times 20$ & $\ldots$ & $\ldots$ & $\ldots$ & $\ldots$ & $70 \mu \mathrm{m}:<5.1(3 \sigma)$ \\
\hline
\end{tabular}

Notes.

${ }^{\mathrm{a}}$ The first value is frame time in seconds and the second value is the number of frames.

${ }^{\mathrm{b}} 20$ frames for $3.6 \mu \mathrm{m}$ and $5.8 \mu \mathrm{m}$ and 26 frames for $4.5 \mu \mathrm{m}$ and $8.0 \mu \mathrm{m}$. 

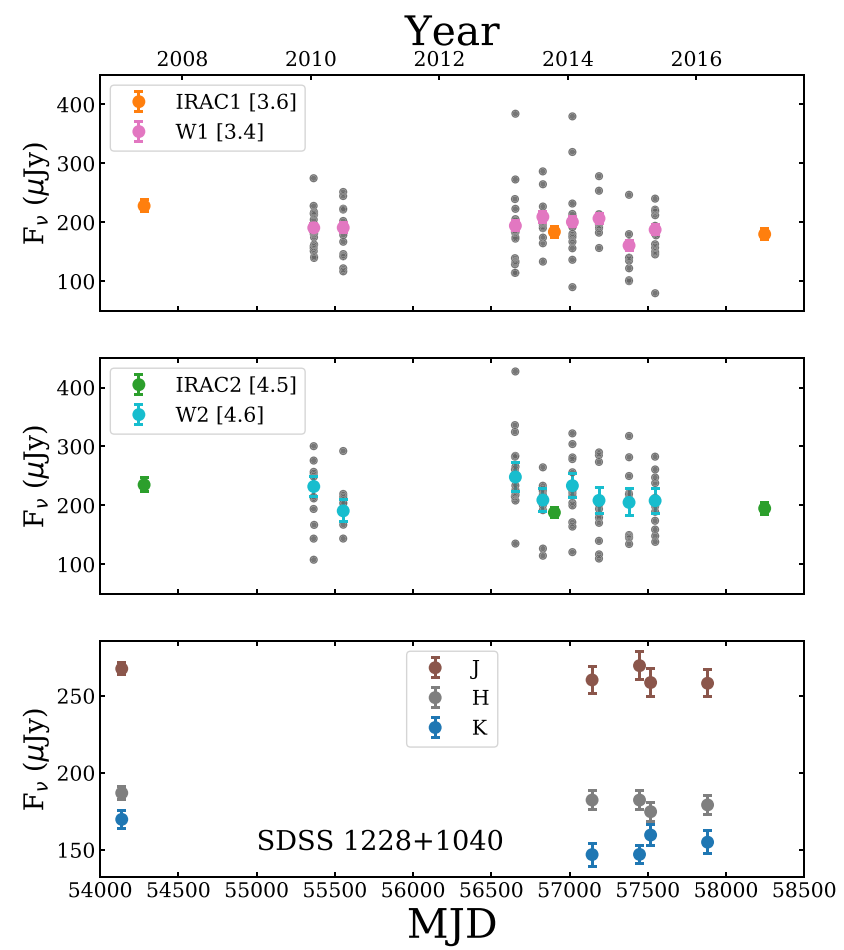
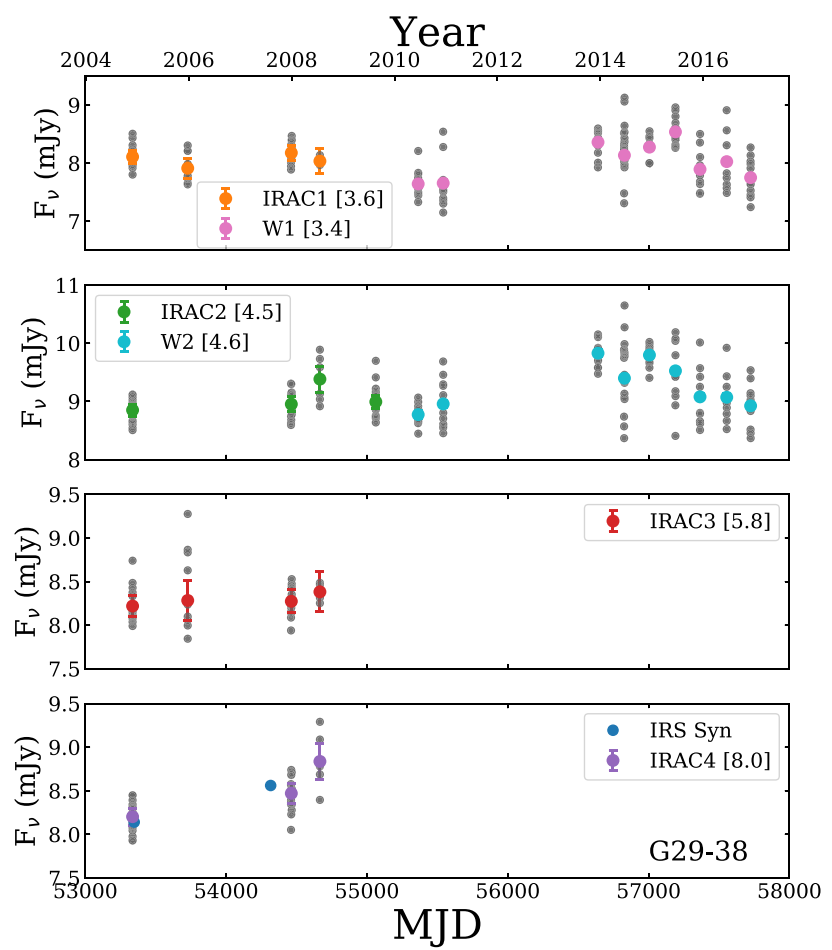

Figure 1. Infrared fluxes of SDSS $1228+1040$ and G29-38. The gray dots represent individual measurements while the colored dots with error bars represent the average flux. For SDSS $1228+1040$, a $20 \%(3 \sigma)$ flux drop is detected between the first two IRAC epochs and a $13 \%(2.7 \sigma)$ drop is detected at K band between the first two UKIRT observations. For G29-38, there are some low level variabilities. The synthetic photometry from IRS spectra are also presented at the [8.0] panel.

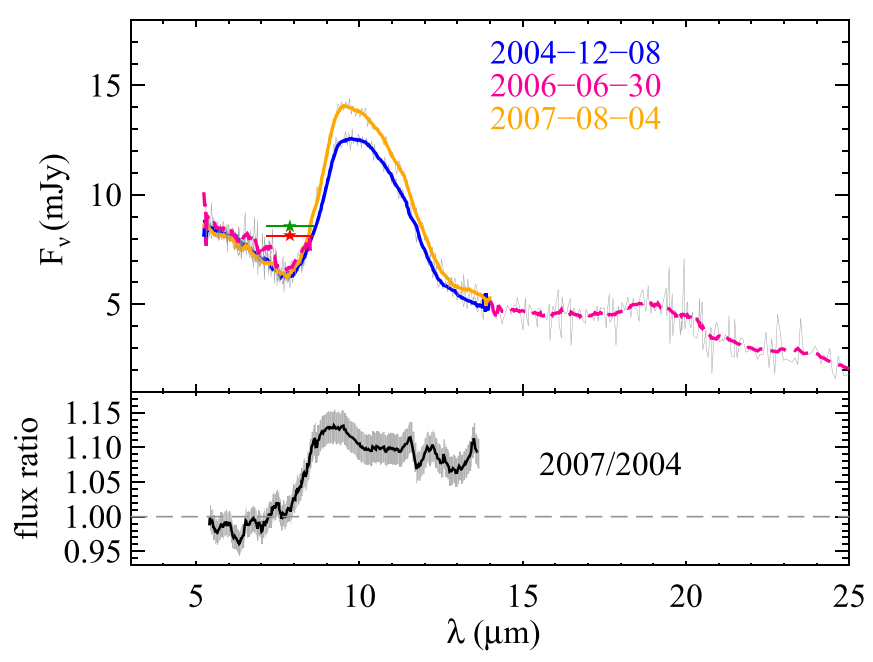

Figure 2. Upper panel shows the smoothed IRS 5-15 $\mu \mathrm{m}$ spectra for G29-38 taken in 2004 (blue, 5.2-14.5 $\mu \mathrm{m}$ ), 2006 (pink, 5.2-8.7 $\mu \mathrm{m}$ and 19.4-38 $\mu \mathrm{m}$ ), and 2007 (yellow, 5.2-14.5 $\mu \mathrm{m}$ ). For comparison, the unsmoothed spectra are also shown as thin gray lines. The [8.0] IRS synthesized photometry is shown as the star symbols (red for 2004, and dark green for 2007) with horizontal bars representing the half bandpass. The bottom panel shows the flux ratio between 2004 and 2007.

listed in Table 1 (Dufour et al. 2017). To match the SDSS rgiz photometry, an additional scaling factor of 0.92 is applied. To calculate the excess infrared flux, we subtract the white dwarf flux from the measured flux, assuming a $2 \%$ uncertainty in the white dwarf flux, as shown in Figure 4.

For the fit, we consider both the IRAC fluxes and the average value of the JHK photometry. For the 2007 observation, we fix the inclination at $70^{\circ}$, which is the same as the gas debris. We performed a chi-squared minimization and the best-fit parameters are listed in Table 6. With all four IRAC points, the disk parameters are well constrained. However, for the 2014 and 2018 data, there are many parameters that could fit the data given the lack of longer wavelength observations. Therefore we started with the best-fitted parameters for the 2007 data and vary one parameter at a time. The change of the infrared flux can be modeled by a decrease of the total emitting area, either by a change of the disk inclination (model 2A) or a change of the disk radius and correspondingly a change in the dust temperature (models $2 \mathrm{~B}$ and $2 \mathrm{C}$ ). The opaque disk model cannot match the high MIPS flux, either because of the change of disk flux between the IRAC and MIPS observations or the presence of an optically thin layer, similar to the case for G2938 , as discussed in the next section.

Manser et al. (2016b) found that the multiyear variation of the emission lines is consistent with precession of an eccentric pattern under general relativity. Note that such precession could not explain the infrared variability assuming the disk is geometrically thin and optically thick. However, if the disk is not geometrically thin, as suggested by recent studies (Kenyon \& Bromley 2017), the infrared flux change might be explained by the obscuring of dust materials from a different part of the disk. This model is beyond the scope of current work and will be explored in a future study.

\section{2. $G 29-38$}

\subsubsection{Stellar Pulsation}

Through 10 years optical photometric monitoring of G29-38, Kleinman et al. (1998) identified 19 pulsation modes with periods between 100 and $1300 \mathrm{~s}$, and not all the modes are excited at the same time. Although the white dwarf pulsation is negligible in the infrared, the flux of the dust disk would change as it reprocesses the star light. To study the effect of 
Table 4

Near-infrared Photometry of SDSS J1228+1040

\begin{tabular}{lcccc}
\hline \hline UT Date & $J(\mathrm{mag})$ & $H(\mathrm{mag})$ & $K(\mathrm{mag})$ & Ref \\
\hline 2007 Feb 7 & $16.893 \pm 0.016$ & $16.841 \pm 0.023$ & $16.425 \pm 0.038$ & $16.582 \pm 0.045$ \\
2015 May 2 & $16.923 \pm 0.017$ & $16.868 \pm 0.017$ & $16.581 \pm 0.027$ & UKIDSS \\
2016 Feb 29 & $16.885 \pm 0.016$ & $16.868 \pm 0.020$ & $16.492 \pm 0.034$ \\
2016 May 10 & $16.930 \pm 0.020$ & $16.914 \pm 0.019$ & $16.524 \pm 0.039$ \\
2017 May 9 & $16.932 \pm 0.018$ & $16.887 \pm 0.019$ & this & thork \\
& & & this & this work \\
\hline
\end{tabular}

white dwarf pulsation on the dust disk, time-series infrared observations of G29-38 have been performed (Graham et al. 1990; Reach et al. 2009). A $190 \mathrm{~s}$ period was identified with an amplitude of $2.5 \%$ at $\mathrm{K}$ band and $4 \%$ at [3.6]; interestingly, this period is not detected in the simultaneous optical light curve. Both Graham et al. (1990) and Reach et al. (2009) postulate that the $190 \mathrm{~s}$ period of the dust disk is induced from pulsations with temperature changes along the latitude (e.g., $m=0$ modes), which has a net temperature change on the dust disk; while the other modes are confined to regions perpendicular to the dust disk (e.g., sectoral, $m=1$ modes) and they cause no net temperature change on the dust disk.

The largest pulsation amplitude is $\pm 5 \%$ in the optical, which corresponds to a $2 \%$ white dwarf temperature change (Kleinman et al. 1998). The average stellar temperature is $11,240 \mathrm{~K}$ (see Table 1), suggesting that the stellar temperature can be as low as $11,015 \mathrm{~K}$ in the low state, but as high as $11,465 \mathrm{~K}$ in the high state. Because the disk is directly heated by the white dwarf, the temporal change of the $10 \mu \mathrm{m}$ feature might be caused by the different stellar temperatures due to pulsation. In the following subsection, we will first introduce our simple two-component model that can fit the disk SED, and then explore the likely change in the observed SED due to two different states of stellar heating.

\subsubsection{A Two-component Disk Model}

As has been explored by Reach et al. (2009), there are many different flavors of disk models that can fit G29-38's SED including a very elaborate mineralogical model. Generally, there are two major parts in the models for white dwarf disks: a component that contributes most of the featureless mid-infrared flux, and a component that is responsible for the solid-state feature. We used the geometrically thin and optically thick disk model (same as for SDSS J1228+1040 in Section 3.1) to represent the featureless component. The prominent solid-state features in the observed mid-infrared spectra most likely come from the dust emission in an optically thin part of the disk, which could be the part of a warped disk (e.g., GD 362, Jura et al. 2007) or the outer part of a wedge-like disk (e.g., Reach et al. 2009). Ideally, one should use a radiative transfer model to self-consistently compute the disk model for both parts; however, such a model has many parameters that current, nonsimultaneously obtained multiwavelength data cannot constrain. A full exploration of various parameters using a radiative transfer model will be presented in a future study. Our main goal here is to understand the temporal change of the $10 \mu \mathrm{m}$ feature. Therefore, we model the G29-38 disk as a twocomponent disk model.

We assume that the optically thin part of the disk is the topmost and outermost layer of the optically thick disk. Both the optically thin and thick disks share the same inner radius, while their outer radii can be different. A larger outer radius for the optically thin disk (hence cooler material) is necessary to account for the midinfrared flux. We assume that the dust is sublimated to gas when its thermal equilibrium temperature reaches the dust sublimation temperature. In this case, the flux contribution from these grains is set to zero. The dust in the innermost region of white dwarf disks can reach 2500-3000 K, which is generally higher than the same material in a protoplanetary disk due to the presence of metallic gas (Rafikov \& Garmilla 2012). We set the dust sublimation temperature to be $2000 \mathrm{~K}$ for silicates, and compute thermal equilibrium dust temperatures for a range of grain sizes and their resultant emission using the program developed for modeling debris disks around main-sequence stars (e.g., $\mathrm{Su}$ et al. 2015). Since the exact mechanism that creates the dust grains in white dwarf disks is unknown, we adopt a standard particle size distribution with a power index of -3.5 , resulting from collisional cascades (Dohnanyi 1969). Silicate grains larger than a few $\mu \mathrm{m}$ in size contribute little to the strength of the $10 \mu \mathrm{m}$ feature; therefore, we can only constrain the largest grain size that is required to fit the shape of the feature. Larger grains will contribute the underlying continuum, which are part of the optically thick disk and remain unconstrained under the assumption of the two-component model. There are four free parameters for the optically thin disk: the outer radius of the disk, the minimum and maximum grain sizes, and the total dust mass (assuming a density of $3.5 \mathrm{~g} \mathrm{~cm}^{-3}$ ).

Given the number of free parameters, there is a range of disk parameters that can fit the G29-38 spectrum satisfactorily. By using olivine grains $\left(\mathrm{Mg}_{0.4} \mathrm{Fe}_{0.6} \mathrm{SiO}_{4}\right.$, Dorschner \& Henning $1995)$ and the low state of the stellar temperature $\left(T_{\mathrm{wd}, \text { low }}=\right.$ $11,015 \mathrm{~K}$ ), we are able to obtain a very good match in the $10 \mu \mathrm{m}$ feature for the 2004 epoch, and a reasonable fit in the midinfrared SED; however, the fit in the $20 \mu \mathrm{m}$ region is less good, as shown in the left panel of Figure 5. One possibility for this poor match is that the 2006 IRS LL spectrum was obtained at a different epoch from the 2004 SL spectra. A more likely explanation is that the optical constants for the pure olivine measured in the laboratory are not exactly matched to the properties of astronomical material. We will explore different grain composition in Section 3.2.3. It is worth pointing out that in the two-component model, the optically thin part also contributes to the continuum flux between 3 and $8 \mu \mathrm{m}$. For SDSS J1228+1040, due to the lack of longer wavelength data, we did not consider this component. The optically thin component is likely to be present due to the high IRAC [8.0] and MIPS fluxes. If such a component is common in dusty white dwarfs, then it could potentially explain the changes observed in SDSS J1228+1040 and SDSS J0959-0200. Future high-quality infrared spectroscopic observations will be crucial for studying the disk structure around white dwarfs. 
Table 5

Near-infrared Photometry of G29-38

\begin{tabular}{lcccc}
\hline \hline UT Date & $J(\mathrm{mag})$ & $H(\mathrm{mag})$ & $K$ (mag) & Reference \\
\hline 2000 Aug 7 & $13.132 \pm 0.026$ & $13.075 \pm 0.022$ & $12.689 \pm 0.029$ & $2 \mathrm{MASS}$ \\
2009 Jun 21 & $13.123 \pm 0.002$ & $13.025 \pm 0.002$ & $12.552 \pm 0.003$ & UKIDSS \\
2015 Aug 9 & $13.127 \pm 0.010$ & $13.038 \pm 0.010$ & $12.595 \pm 0.013$ & this work \\
2016 Jul 6 & $13.141 \pm 0.010$ & $13.042 \pm 0.010$ & $12.623 \pm 0.020$ & this work \\
\hline
\end{tabular}

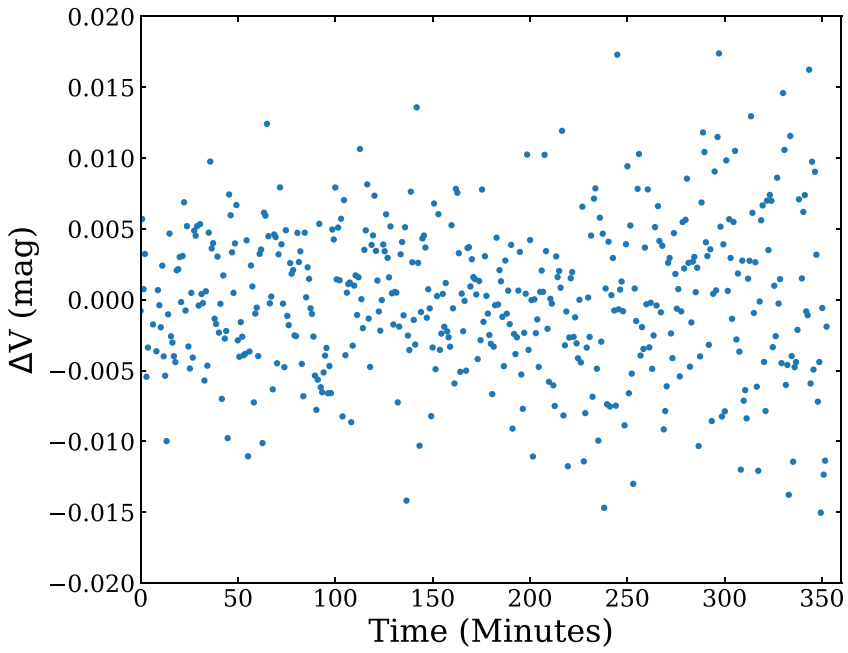

Figure 3. Optical monitoring of SDSS J1228+1040 from the 61 inch telescope. No optical variability is detected and the standard deviation of the light curve is $0.006 \mathrm{mag}$. The scatter toward the end of the observations is due to the increasing airmass of the target.
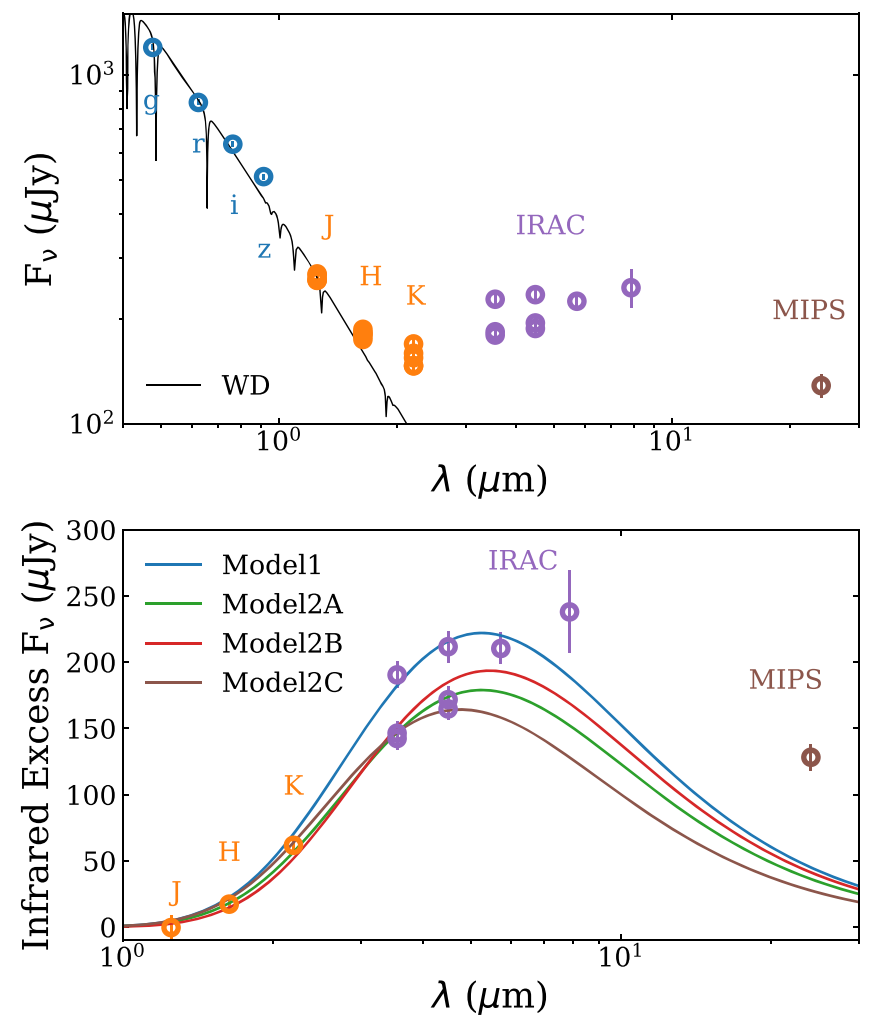

Figure 4. SED fits for SDSS J1228+1040. The photometry points are from the SDSS (griz), UKIRT (JHK), and Spitzer (IRAC and MIPS). The top panel shows the entire SED while the bottom panel is the excess infrared flux. The parameters for the models are listed in Table 6 . The change of the infrared fluxes can either be modeled by a change of the inclination, inner disk radius, or outer disk radius.
Table 6

Fitting Parameters for SDSS J1228+1040

\begin{tabular}{lcccc}
\hline \hline & Model 1 & Model 2A & Model 2B & Model 2C \\
\hline Inner radius $R_{\text {in }}$ & $27 R_{\mathrm{wd}}$ & $27 R_{\mathrm{wd}}$ & $30 R_{\mathrm{wd}}$ & $27 R_{\mathrm{wd}}$ \\
Outer radius $R_{\text {out }}$ & $63 R_{\mathrm{wd}}$ & $63 R_{\mathrm{wd}}$ & $63 R_{\mathrm{wd}}$ & $49 R_{\mathrm{wd}}$ \\
Inclination $i$ & $70^{\circ}$ & $74^{\circ}$ & $70^{\circ}$ & $70^{\circ}$ \\
$\chi^{2}$ & 7.2 & 2.7 & 10.6 & 0.8 \\
\hline
\end{tabular}

Note. Model 1 is the best-fit parameters for the 2007 IRAC data ([3.6], [4.5], [5.8], and [8.0]), while Model 2A, 2B, and 2C are for the 2014 and 2018 IRAC data ([3.6] and [4.5]).

We now explore whether the change in the $10 \mu \mathrm{m}$ feature could be caused by the change of the stellar heating. With our base low-state model parameter determined, we recompute the dust equilibrium temperatures using the high stellar temperature $\left(T_{\text {wd,high }}=11,465 \mathrm{~K}\right)$, and derive the output SED by using the same disk parameters that fit the 2004 spectrum. Increasing the heating power does increase the overall disk emission because the grains are at slightly higher temperatures, and the changes are slightly different between the two parts of the disk. As shown in the right panel of Figure 5, the net effect is that the change from stellar pulsation is wavelength dependent. With the fixed disk parameters (location, grain sizes and mass), we expect the pulsation-induced change in the disk emission to be higher at the $2-7 \mu \mathrm{m}$ region than that of the $10 \mu \mathrm{m}$ region. The maximum pulsation-induced variability $(\approx 15 \%)$ occurs at $3 \mu \mathrm{m}$, while it is mostly below $10 \%$ at other wavelengths. We also test a more extreme case with a larger temperature swing of $\pm 1000 \mathrm{~K}$ for the star. The change is more dramatic (a larger flux ratio overall) in this case, but the relative wavelength-dependent trend remains the same. From these calculations, we conclude that stellar pulsation is likely to be responsible for the low level variability observed in the $K_{\mathrm{s}}$ and IRAC bands (discussed in Sections 2.1 and 2.4). However, the same mechanism is unlikely to explain the flux change around $10 \mu \mathrm{m}$ because we see no flux change in the IRS 5-7 $\mu \mathrm{m}$ region between all three IRS epochs. In addition, the integration times of the IRS SL spectra are $366 \mathrm{~s}$, a factor of two longer than the pulsation period of $190 \mathrm{~s}$ found in the infrared light curve. As a result, any pulsation effect on heating the dust is likely to be averaged out for the IRS observations. Therefore, the change in the $10 \mu \mathrm{m}$ feature most likely comes from the intrinsic change of the disk parameters in the optically thin part of the disk.

\subsubsection{Variability from an Increase of Dust Mass}

We now proceed to explore the possible changes in the disk parameters that could be responsible for the $10 \mu \mathrm{m}$ feature variation. Since the change of stellar effective temperature is unlikely to be the main reason, we fix the stellar effective temperature to be at the average temperature of $11,240 \mathrm{~K}$ for 

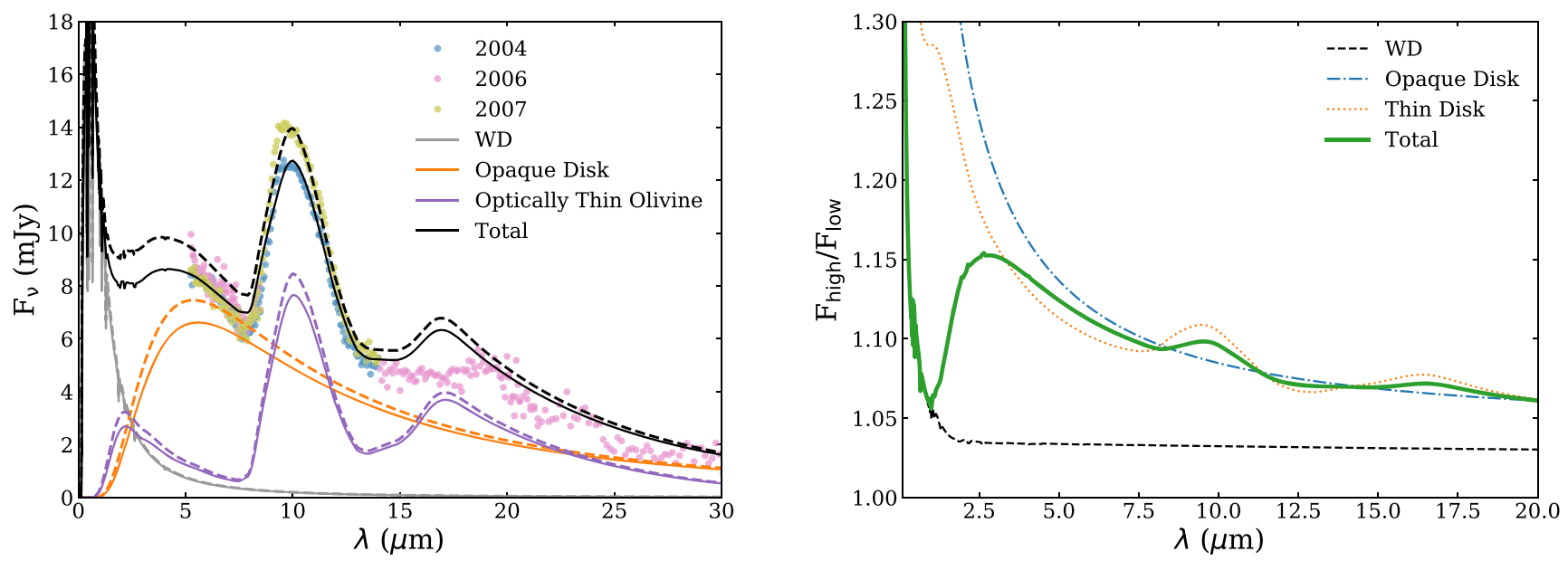

Figure 5. Two-component disk model with $T_{\mathrm{wd}, \text { low }}=11,015 \mathrm{~K}$ and $T_{\mathrm{wd}, \text { high }}=11,465 \mathrm{~K}$. Left panel: the dashed line represents the high temperature state, while the solid line represents the low temperature state. The stellar temperature is the only difference and the disk parameters are all kept the same. Right panel: the black, blue, orange, and green lines represent the ratio of the highest and lowest flux for the white dwarf, optically thick disk, optically thin disk, and the total flux, respectively. If the $10 \mu \mathrm{m}$ flux change comes from pulsation, then we would expect a flux change of at least $10 \%$ around 5-7 $\mu \mathrm{m}$, which was not observed.
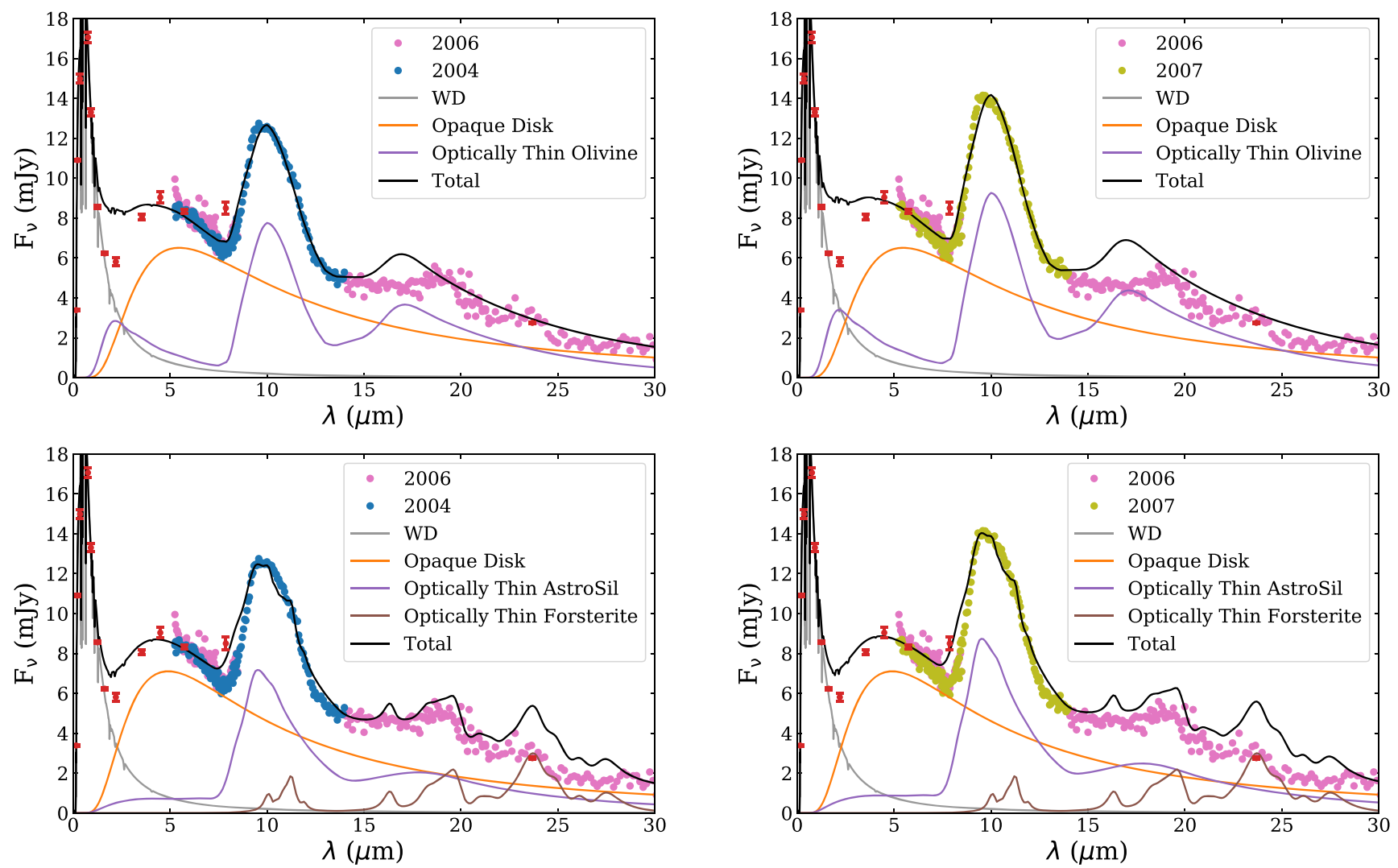

Figure 6. Two-component disk models of G29-38 for fitting the Spitzer/IRS data. The top row shows models using olivine composition, and the bottom shows the ones using a combination of astronomical amorphous and crystalline silicates. The difference between the 2004 and 2007 IRS data can be explained by an increase of dust mass.

the rest of our modeling for simplicity. In addition to the olivine grains used in the previous section, we also consider astronomical silicates (Laor \& Draine 1993) for the grain composition. Similarly, we first derive the model parameters using the 2004 IRS spectrum for both compositions. Using the astronomical silicates, as shown in Figure 6, the $10 \mu \mathrm{m}$ feature shape is not a good match at all (a shift in the peak of the feature), but the fit to the $17 \mu \mathrm{m}$ region is much better compared to the one using olivine grains. In fact, adding a small amount of crystalline silicates (forsterite, $\mathrm{Mg}_{2} \mathrm{SiO}_{4}$, Koike et al. 2003) improves the match to the red side of the $10 \mu \mathrm{m}$ feature and the overall features in the IRS LL spectrum, consistent with the finding by Reach et al. (2009). Satisfactory fits can be achieved by using either olivine or astronomical silicates with a small amount of forsterite.

As discussed in Section 3.2.2, there is a wide range of parameters that can give reasonable fits to the G29-38 spectrum. We are mostly interested in the cause for the change 
Table 7

Two-component Disk Parameters for G29-38

\begin{tabular}{|c|c|c|c|c|c|}
\hline Component & Description & \multicolumn{2}{|c|}{ Olivine Composition } & \multicolumn{2}{|c|}{ Astronomical Silicates ${ }^{\mathrm{a}}$} \\
\hline \multirow[t]{3}{*}{ Opaque disk } & Inner radius $R_{\text {in }}$ & \multicolumn{2}{|c|}{$10 R_{\mathrm{wd}}$} & \multicolumn{2}{|c|}{$8 R_{\mathrm{wd}}$} \\
\hline & Outer radius $R_{\text {out }}$ & \multicolumn{2}{|c|}{$28 R_{\mathrm{wd}}$} & \multicolumn{2}{|c|}{$25 R_{\mathrm{wd}}$} \\
\hline & Inclination $i$ & \multicolumn{2}{|c|}{$30^{\circ}$} & \multicolumn{2}{|c|}{$30^{\circ}$} \\
\hline \multirow[t]{4}{*}{ Optically thin disk } & Grain sizes & \multicolumn{2}{|c|}{$0.5-5.0 \mu \mathrm{m}$} & \multicolumn{2}{|c|}{$0.1-3.0 \mu \mathrm{m}$} \\
\hline & Outer radius & \multicolumn{2}{|c|}{$167 R_{\mathrm{wd}}$} & \multicolumn{2}{|c|}{$834 R_{\mathrm{wd}}$} \\
\hline & Dust mass & 2004 epoch & 2007 epoch & 2004 epoch & 2007 epoch \\
\hline & & $\overline{1.1 \times 10^{18} \mathrm{~g}}$ & $\overline{1.4 \times 10^{18} \mathrm{~g}}$ & $\overline{4.0 \times 10^{18} \mathrm{~g}}$ & $\overline{4.8 \times 10^{18} \mathrm{~g}}$ \\
\hline
\end{tabular}

Note.

${ }^{\mathrm{a}}$ with a small amount $\left(1.6 \times 10^{18} \mathrm{~g}\right)$ of sub $\mu \mathrm{m}$ forestite grains at a fixed dust temperature of $250 \mathrm{~K}$.

in the $10 \mu \mathrm{m}$ feature, so we deliberately fix most of the parameters in the models, and only vary the amount of dust in the optically thin disk when fitting the 2007 epoch of the IRS spectrum. The parameters used for the fits are given in Table 7. As shown in Figure 6, the difference between the 2004 and 2007 spectra can be explained by an increase of $\approx 20 \%$ in the dust mass for both compositions. We further explore whether the change in grain sizes in the optically thin disk could explain the difference seen in the two epochs. Within the uncertainty, the 2007 IRS spectrum is also consistent with a model using a slightly smaller size range of olivine grains and an increase of dust mass by $5 \%$. Under the two-component disk model, we conclude that the most likely cause of variability in the IRS spectra is due to an increase $(5 \%-20 \%)$ in the mass of small grains.

Note that the dust mass derived from our optically thin disk model is lower than the value derived by Reach et al. (2009), where a total of $2 \times 10^{19} \mathrm{~g}$ is needed with a grain size of $0.1-10 \mu \mathrm{m}$. In addition to the small difference in grain sizes, the majority of the difference comes from the fact that the mass we estimate does not include the optically thick part of the disk, where its contribution was accounted for as the emission from the carbon-like grains in Reach et al. (2009).

\section{Implications}

It is exciting that we are detecting temporal variation of dust disks around white dwarfs. SDSS J1228+1040, together with SDSS J0959-0200, seem to belong to the same category. Their [3.6] and [4.5] fluxes dropped by 20\%-30\% within a few years and then mostly remain the same afterward. For G29-38, the $10 \mu \mathrm{m}$ feature has increased by $10 \%$ within three years while the 5-7 $\mu \mathrm{m}$ fluxes remained the same. Because of the limited coverage, we do not know whether it is a sudden or gradual drop in the infrared flux or a temporal increase followed by a drop. We also do not know yet the long-term trend of these variations. In addition, SDSS J1228+1040 and SDSS J0959-0200 both display significant amounts of circumstellar gas.

For G29-38, the increase of $10 \mu \mathrm{m}$ flux is most likely caused by an increased dust mass of $\sim 10^{17} \mathrm{~g}$ in the optically thin component. A continuous change between the two IRS observations (2004 December 8 and 2007 August 4) would correspond to a dust production rate of $10^{9} \mathrm{~g} \mathrm{~s}^{-1}$, which is comparable to the mass accretion rate of $7 \times 10^{8} \mathrm{~g} \mathrm{~s}^{-1}$ observed in the atmosphere of G29-38 (Xu et al. 2014).

For SDSS J1228+1040, under the opaque disk assumption, the change in the 3-8 $\mu \mathrm{m}$ flux can be explained by a change in the disk inclination or a decrease of disk surface area by $8 \%-48 \%$. We can estimate the lower limit of the mass of the opaque disk to be $\pi\left(R_{\text {out }}^{2}-R_{\text {in }}^{2}\right) \times 2 h \times \rho \sim$ $10^{23} \mathrm{~g}$ assuming a scale height $h$ of $1 \mathrm{~cm}$ and a density $\rho$ of $3 \mathrm{~g} \mathrm{~cm}^{-3}$. Such a decrease in mass over the two IRAC observations separated by 7 years would require a mass-loss rate of $4 \times 10^{13}-2 \times 10^{14} \mathrm{~g} \mathrm{~s}^{-1}$. This rate is significantly higher than the mass accretion rate of $6 \times 10^{8} \mathrm{~g} \mathrm{~s}^{-1}$ derived from its atmospheric pollution (Gänsicke et al. 2012).

Here, we explore some mechanisms that could lead to the observed infrared variability.

\subsection{Tidal Disruption Event}

As discussed in Jura (2008), tidal disruption of a massive minor planet could disrupt the pre-existing disk. Because the incoming body is likely to have a different orbital inclination, a new dust disk will eventually be formed at a different orbital inclination. The final infrared flux could be either higher or lower than the previous one. In this scenario, a significant amount of dust will be evaporated to produce circumstellar gas, which could explain the presence of the calcium infrared triplet observed around SDSS J1228+1040 and SDSS J0959-0200. Because the emission lines are optically thick, it is possible for the total amount of gas to change without the strength of the emission line changing. However, from the smooth change of the calcium infrared triplet lines of SDSS J1228+1040 from 2003 to 2015 (Manser et al. 2016b), there is little evidence for a tidal disruption event.

In order for an incoming minor planet to disrupt the preexisting dust disk, it needs to be significantly more massive than the dust disk, which is estimated to be $10^{23} \mathrm{~g}$. Given that current infrared monitoring of dusty white dwarfs is sparse, the chance of finding variable systems is slim. However, finding at least two infrared variable dusty white dwarfs (SDSS J1228 +1040 and SDSS J0959-0200) out of a total of 43 systems over 7 years gives an upper limit of one major tidal disruption event (mass $>10^{23} \mathrm{~g}$, diameter $>200 \mathrm{~km}$ ) every 140 years, which is significantly shorter than the frequency of tidal disruption events derived from dynamical simulations (Veras 2016; Mustill et al. 2018). In addition, with such an energetic tidal disruption event, the infrared excess is likely to display a temporary increase, which needs to fit in this already extremely short timescale.

Apart from these major tidal disruption events, it has been suggested that accretion of small planetesimals $(<35 \mathrm{~km}$, $\sim 10^{19} \mathrm{~g}$ ) is nearly continuous (Wyatt et al. 2014). The increase of the $10 \mu \mathrm{m}$ feature in G29-38 could come from continuous accretion of small planetesimals, if they all became small dust. 


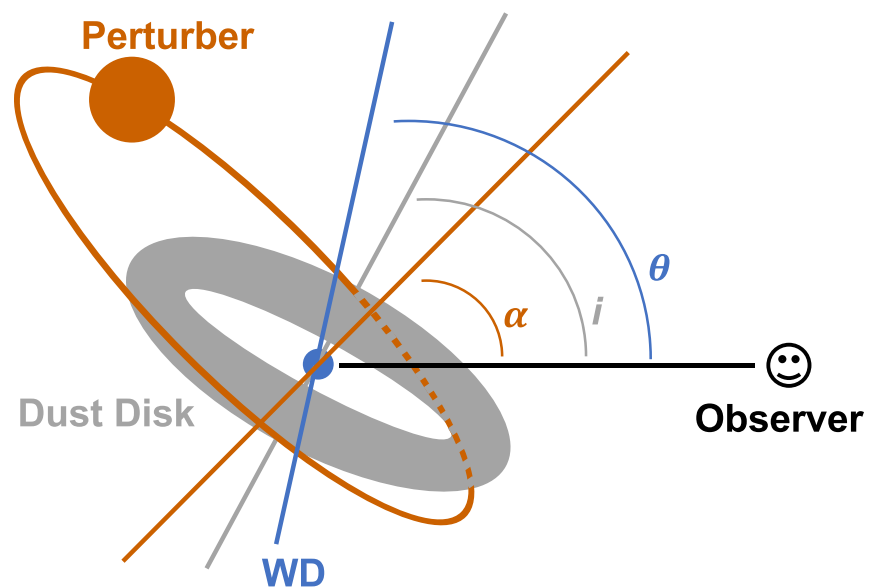

Figure 7. Cartoon illustration of a white dwarf, a dust disk, and an unseen perturber. Their orbital planes are not aligned and $\alpha, i, \theta$ are defined as the angle between our line of sight and the orbital plane of the perturber, the orbital plane of the dust disk, and the white dwarf rotation axis (pulsation axis). Note that the figure is not to scale.

An increase of $10^{17} \mathrm{~g}$ could come from an object of $4 \mathrm{~km}$ in diameter.

\subsection{External Perturbation}

Here, we explore the response of the dust disk in the presence of an external perturber on an inclined orbit. By studying the pulsation light curve of G29-38, Montgomery (2005) derived the rotation axis (pulsation axis) $\theta$ to be $65^{\circ}$ (see Figure 7 for a cartoon illustration), which is comparable to $55^{\circ}$ derived from comparing the amplitudes of the harmonics (Thompson et al. 2010). The white dwarf rotation axis might not be aligned with the axis $i$ of the opaque disk, which is derived to be $30^{\circ} .13$ This is not surprising given tidal disruption is a dynamical process and the inclination of the orbits of the minor planets can vary significantly. An external perturber that recently arrived on an orbit that is inclined with respect to the dust disk can perturb the latter and possibly cause the infrared variability. These perturbers are expected to be the catalysts for the pollution of white dwarf atmospheres (Debes \& Sigurdsson 2002; Veras \& Gänsicke 2015).

To quantitatively estimate the effect of an external perturber on the dust disk, we ran a basic suite of $N$-body simulations for the SDSS J1228+1040 system. We modeled the disk as a series of five coplanar circular rings each containing 30 massless particles uniformly distributed in azimuth. The location of the rings were at 20,40,60,80, and $100 R_{\mathrm{wd}}$ away from the center of the white dwarf. We carried out the simulations with a modified version of the Mercury integration package (Chambers 1999), which allowed us to incorporate the effects of general relativity. This exercise is in the same spirit as that in Manser et al. (2016b), except here the perturber's orbital plane is not aligned with the dust disk $(\alpha \neq i$ in Figure 7).

We found that the degree of perturbation is strongly dependent on the perturber's mass and orbital properties (semimajor axis, eccentricity, inclination). We focus on an extreme case with a $10 M_{\mathrm{J}}$ perturber at a semimajor axis of $5 \mathrm{au}$, while the pericenter of the perturber's orbit (ranging from $100 R_{\mathrm{wd}}$ to $\left.200 R_{\mathrm{wd}}\right)$ and orbital inclination $(\alpha-i \mid$ ranging

\footnotetext{
13 The inclination of the opaque disk also depends on the contribution of the optically thin component in 3-8 $\mu \mathrm{m}$ (see Section 3.2.2).
}

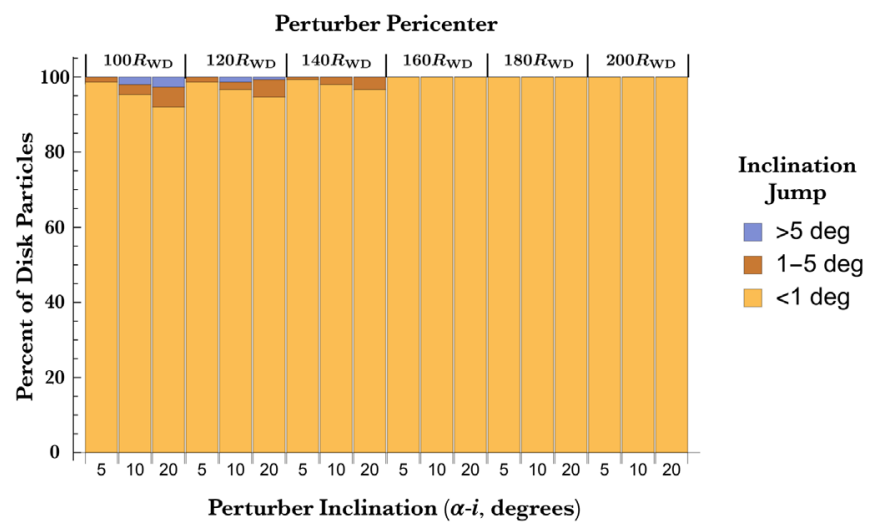

Figure 8. Excitations of disk particles at different orbital radii as a function of perturber orbital pericenter and perturber inclination $|\alpha-i|$. This simulation is for one pericenter passage of a $10 M_{\mathrm{J}}$ planet with a semimajor axis of $5 \mathrm{au}$. The plot suggests that even in the most optimistic case, only a small part of the disk can reach an inclination change larger than $1^{\circ}$.

from $5^{\circ}$ to $20^{\circ}$ ) are taken at different values. The result from one pericenter passage is shown in Figure 8. The disk is perturbed the most when the perturber has a pericenter closest to the disk edge with a large inclination angle. However, even in the most optimistic case, only a small fraction of the disk can be perturbed by more than $1^{\circ}$ after one pericenter passage, which is not sufficient for the $4^{\circ}$ inclination change required to reproduce the change of the infrared excess.

We consider in our simulation a typical pericenter passage; in reality, the disk would be perturbed every time the perturber passes the pericenter. In addition, our simulations show that with a perturbing object, the disk will no longer remain flat, which is also suggested by numerical studies of dust disks (Kenyon \& Bromley 2017). This change could reduce the optical depth of the dust disk and correspondingly increase the dust temperature, leading to grain sublimation and a drop in the infrared luminosity. Exploring this scenario would require $N$-body simulations over multiple pericenter passages and is beyond the scope of this work.

\subsection{Runaway Accretion}

The infrared variability of SDSS J1228+1040 and SDSS J0959-0200 are very similar and they both display gas emission lines from the calcium infrared triplet. It has been suggested that when there is a strong coupling between the dust and gas, runaway accretion could occur and it can lead to a higher accretion rate than Poynting-Robertson drag can support (Rafikov 2011; Metzger et al. 2012). The evolution of the disk is significantly different from those without gas drag.

For SDSS $\mathrm{J} 1228+1040$, the mass accretion rate supported by Poynting-Robertson drag is $\sim 10^{9} \mathrm{~g} \mathrm{~s}^{-1}$. During runaway accretion, the peak value can reach $\sim 10^{3}$ higher than the rate from Poynting-Robertson drag (Metzger et al. 2012). This higher value is still slightly lower than the derived mass-loss rate of the dust disk $\left(10^{13}-10^{14} \mathrm{~g} \mathrm{~s}^{-1}\right)$. For runaway accretion to occur, there are two main criteria: (I) the dust disk is massive enough ( $\gtrsim 10^{22} \mathrm{~g}$ ); (II) there is a build-up of gas due to sublimation occurring faster than the rate that gas is removed by viscous diffusion (Rafikov 2011). Both SDSS J1228+1040 and SDSS J0959-0200 have strong infrared excesses, which likely originate from a massive disk. Criterion II requires a 
strong gas-solid coupling factor and/or a weak gas viscosity. Although there are a lot of uncertainties in these parameters, it could be satisfied for white dwarf disks (Metzger et al. 2012).

Runaway accretion has not been directly observed in white dwarf disks. An indirect line of evidence is that some of the helium-dominated white dwarfs with circumstellar gas (e.g., WD J0738+1835, Ton 345) have the highest mass accretion rates of all polluted white dwarfs, which is expected from runaway accretion. Once runaway accretion starts, the solid disk will be dissipated in a very short amount of time. Correspondingly, the infrared flux will continue to drop and the mass accretion rate would increase substantially (Metzger et al. 2012). So far, there is no evidence of continued drop in the infrared flux, nor an increase of absorption line strength in the atmosphere (Manser et al. 2016b). Future monitoring would be crucial in assessing the runaway accretion scenario.

\section{Conclusion}

In this paper, we presented infrared variabilities of two white dwarfs with dust disks, SDSS J1228+1040 and G29-38. For SDSS J1228+1040, the IRAC [3.6] and [4.5] fluxes dropped by $20 \%$ within 7 years and remained the same afterward. The general behavior is very similar to the flux drop around another dusty white dwarf WD J0959-0200 (Xu \& Jura 2014) with a further similarity being the appreciable amounts of circumstellar gas around both objects. The flux drop can be explained by either an increase of an inner disk radius, a decrease in the outer disk radius, or a change in the disk inclination, assuming the excess comes from an opaque dust disk.

G29-38 appears to represent a different kind of infrared variability and the flux of the $10 \mu \mathrm{m}$ feature has increased by $10 \%$ within 3 years, while the 5-7 $\mu$ m flux remained the same. We presented a two-component disk model to fit the infrared spectra, and concluded that the change is unlikely to be related to the photospheric pulsation of G29-38 with a static disk. We propose that the most likely cause is an increase in the mass of small grains in the optically thin component.

To explain the observed infrared variability, we explored several scenarios, including tidal disruption events, external perturbation, and runaway accretion. Although continuous tidal disruptions of small planetesimals could explain the increased dust mass in G29-38, no satisfactory scenarios can explain the sudden drop of infrared flux for SDSS J1228+1040 and SDSS J0959-0200.

Looking forward, a self-consistent radiative transfer disk model would be valuable in constraining white dwarf disk parameters. In the future, continued photometric monitoring in the infrared and high-quality infrared spectroscopy from the James Webb Space Telescope will be crucial in constructing a complete picture of dust disks around white dwarfs.

The authors would like to thank the late UCLA Professor Michael Jura for his inspirations and support to study white dwarf disks. We thank S. Kleinman and A. Nitta for helpful discussions about white dwarf pulsation. A.B. acknowledges a Royal Society Dorothy Hodgkin Fellowship. J.O. acknowledges financial support from the ICM (Iniciativa Científica Milenio) via the Núcleo Milenio de Formación Planetaria grant, from the Universidad de Valparaíso, and from Fondecyt (grant 1180395). D.V. gratefully acknowledges the support of the Science and Technology Facilities Council via an Ernest Rutherford Fellowship (grant ST/P003850/1). L.K.R and
T.G.W wish to acknowledge funding from an STFC studentship. The research leading to these results has also received funding from the European Research Council under the European Union's Seventh Framework Programme (FP/ 2007-2013)/ERC Grant Agreement No. 320964 (WDTracer).

This work is based in part on observations made with the Spitzer Space Telescope, which is operated by the Jet Propulsion Laboratory, California Institute of Technology, under a contract with NASA. This publication makes use of data products from the Wide-field Infrared Survey Explorer (WISE), which is a joint project of the University of California, Los Angeles, and the Jet Propulsion Laboratory/California Institute of Technology, funded by the National Aeronautics and Space Administration. This publication also makes use of data products from the Near-Earth Object Wide-field Infrared Survey Explorer (NEOWISE), which is a project of the Jet Propulsion Laboratory/California Institute of Technology. NEOWISE is funded by the National Aeronautics and Space Administration.

Software: IRAF (Tody 1986, 1993), Mercury (Chambers 1999), Matplotlib (Hunter 2007).

\section{ORCID iDs}

Siyi Xu (许偲艺) (iD https://orcid.org/0000-0002-8808-4282

Kate Y. L. Su (1D) https://orcid.org/0000-0002-3532-5580 Johan Olofsson (iD https://orcid.org/0000-0003-4475-3605 Dimitri Veras (D) https://orcid.org/0000-0001-8014-6162 Elizabeth M. Green (1) https://orcid.org/0000-0003-3688-2298 Everett Schlawin (1D https://orcid.org/0000-0001-8291-6490 Thomas G. Wilson (i) https://orcid.org/0000-0001-8749-1962 David J. Wilson (D) https://orcid.org/0000-0001-9667-9449

Boris T. Gänsicke (i) https://orcid.org/0000-0002-2761-3005

\section{References}

Brinkworth, C. S., Gänsicke, B. T., Marsh, T. R., Hoard, D. W., \& Tappert, C. 2009, ApJ, 696, 1402

Chambers, J. E. 1999, MNRAS, 304, 793

Debes, J. H., \& Sigurdsson, S. 2002, ApJ, 572, 556

Debes, J. H., Walsh, K. J., \& Stark, C. 2012, ApJ, 747, 148

Dennihy, E., Clemens, J. C., Dunlap, B. H., et al. 2018, ApJ, 854, 40

Dohnanyi, J. S. 1969, JGR, 74, 2531

Dorschner, J., \& Henning, T. 1995, A\&ARv, 6, 271

Dufour, P., Blouin, S., Coutu, S., et al. 2017, in ASP Conf. Ser. 509, 20th European White Dwarf Workshop, ed. P.-E. Tremblay, B. Gaensicke, \& T. Marsh (San Francisco, CA: ASP), 3

Farihi, J. 2016, NewAR, 71, 9

Farihi, J., Wyatt, M. C., Greaves, J. S., et al. 2014, MNRAS, 444, 1821

Fazio, G. G., Hora, J. L., Allen, L. E., et al. 2004, ApJS, 154, 10

Gaia Collaboration, Brown, A. G. A., Vallenari, A., et al. 2018, A\&A, 616, A1 Gänsicke, B. T., Aungwerojwit, A., Marsh, T. R., et al. 2016, ApJL, 818, L7

Gänsicke, B. T., Koester, D., Farihi, J., et al. 2012, MNRAS, 424, 333

Gänsicke, B. T., Koester, D., Marsh, T. R., Rebassa-Mansergas, A., \& Southworth, J. 2008, MNRAS, 391, L103

Gänsicke, B. T., Marsh, T. R., Southworth, J., \& Rebassa-Mansergas, A. 2006, Sci, 314, 1908

Gary, B. L., Rappaport, S., Kaye, T. G., Alonso, R., \& Hambschs, F.-J. 2017, MNRAS, 465, 3267

Graham, J. R., Matthews, K., Neugebauer, G., \& Soifer, B. T. 1990, ApJ, 357, 216 Harrison, J. H. D., Bonsor, A., \& Madhusudhan, N. 2018, MNRAS, 479, 3814 Hollands, M. A., Gänsicke, B. T., \& Koester, D. 2018, MNRAS, 477, 93 Houck, J. R., Roellig, T. L., van Cleve, J., et al. 2004, ApJS, 154, 18 Hunter, J. D. 2007, CSE, 9, 90 Jura, M. 2003, ApJL, 584, L91

Jura, M. 2008, AJ, 135, 1785

Jura, M., Farihi, J., Zuckerman, B., \& Becklin, E. E. 2007, AJ, 133, 1927

Jura, M., \& Young, E. D. 2014, AREPS, 42, 45

Kenyon, S. J., \& Bromley, B. C. 2017, ApJ, 844, 116 
Kleinman, S. J., Nather, R. E., Winget, D. E., et al. 1998, ApJ, 495, 424

Koester, D., Gänsicke, B. T., \& Farihi, J. 2014, A\&A, 566, A34

Koester, D., Provencal, J., \& Shipman, H. L. 1997, A\&A, 320, L57

Koike, C., Chihara, H., Tsuchiyama, A., et al. 2003, A\&A, 399, 1101

Laor, A., \& Draine, B. T. 1993, ApJ, 402, 441

Lebouteiller, V., Barry, D. J., Spoon, H. W. W., et al. 2011, ApJS, 196, 8

Mainzer, A., Bauer, J., Grav, T., et al. 2011, ApJ, 731, 53

Manser, C. J., Gänsicke, B. T., Koester, D., Marsh, T. R., \& Southworth, J. 2016a, MNRAS, 462, 1461

Manser, C. J., Gänsicke, B. T., Marsh, T. R., et al. 2016b, MNRAS, 455, 4467

McGraw, J. T., \& Robinson, E. L. 1975, ApJL, 200, L89

Metzger, B. D., Rafikov, R. R., \& Bochkarev, K. V. 2012, MNRAS, 423, 505

Montgomery, M. H. 2005, ApJ, 633, 1142

Mustill, A. J., Veras, D., \& Villaver, E. 2014, MNRAS, 437, 1404

Mustill, A. J., Villaver, E., Veras, D., Gänsicke, B. T., \& Bonsor, A. 2018, MNRAS, 476, 3939

Nordhaus, J., \& Spiegel, D. S. 2013, MNRAS, 432, 500

Rafikov, R. R. 2011, MNRAS, 416, L55

Rafikov, R. R., \& Garmilla, J. A. 2012, ApJ, 760, 123

Reach, W. T., Kuchner, M. J., von Hippel, T., et al. 2005, ApJL, 635, L161

Reach, W. T., Lisse, C., von Hippel, T., \& Mullally, F. 2009, ApJ, 693, 697

Redfield, S., Farihi, J., Cauley, P. W., et al. 2017, ApJ, 839, 42

Rieke, G. H., Young, E. T., Engelbracht, C. W., et al. 2004, ApJS, 154, 25

Shulov, O. S., \& Kopatskaya, E. N. 1974, Ap, 10, 72

Sierchio, J. M., Rieke, G. H., Su, K. Y. L., \& Gáspár, A. 2014, ApJ, 785, 33

Smallwood, J. L., Martin, R. G., Livio, M., \& Lubow, S. H. 2018, MNRAS, 480, 57
Stephan, A. P., Naoz, S., \& Zuckerman, B. 2017, ApJL, 844, L16

Su, K. Y. L., Morrison, S., Malhotra, R., et al. 2015, ApJ, 799, 146

Subasavage, J. P., Jao, W.-C., Henry, T. J., et al. 2017, AJ, 154, 32

Thompson, S. E., Montgomery, M. H., von Hippel, T., et al. 2010, ApJ, 714, 296

Tody, D. 1986, Proc. SPIE, 627, 733

Tody, D. 1993, adass II, 52, 173

Tremblay, P.-E., Bergeron, P., \& Gianninas, A. 2011, ApJ, 730, 128

Vanderburg, A., Johnson, J. A., Rappaport, S., et al. 2015, Natur, 526, 546

Veras, D. 2016, RSOS, 3, 150571

Veras, D., \& Gänsicke, B. T. 2015, MNRAS, 447, 1049

von Hippel, T., Kuchner, M. J., Kilic, M., Mullally, F., \& Reach, W. T. 2007, ApJ, 662, 544

Wilson, D. J., Gänsicke, B. T., Koester, D., et al. 2014, MNRAS, 445, 1878

Wilson, D. J., Gänsicke, B. T., Koester, D., et al. 2015, MNRAS, 451, 3237

Wright, E. L., Eisenhardt, P. R. M., Mainzer, A. K., et al. 2010, AJ, 140, 1868

Wyatt, M. C., Farihi, J., Pringle, J. E., \& Bonsor, A. 2014, MNRAS, 439, 3371

Xu, S., \& Jura, M. 2014, ApJL, 792, L39

Xu, S., Jura, M., Dufour, P., \& Zuckerman, B. 2016, ApJL, 816, L22

Xu, S., Jura, M., Koester, D., Klein, B., \& Zuckerman, B. 2013, ApJL, 766, L18

Xu, S., Jura, M., Koester, D., Klein, B., \& Zuckerman, B. 2014, ApJ, 783, 79 Zuckerman, B., \& Becklin, E. E. 1987, Natur, 330, 138

Zuckerman, B., Koester, D., Reid, I. N., \& Hünsch, M. 2003, ApJ, 596, 477

Zuckerman, B., Melis, C., Klein, B., Koester, D., \& Jura, M. 2010, ApJ, 722,725 